\title{
Battery/ultra-capacitor Hybrid Energy Storage System Used in HEV
}

\author{
Haifang Yu ${ }^{1}$, Rengui Lu ${ }^{2}$, Tiecheng Wang ${ }^{3}$, and Chunbo Zhu ${ }^{4}$ \\ ${ }^{1}$ Department of Electrical Engineering, Harbin Institute of Technology, haifangyu@gmail.com \\ ${ }^{2}$ Department of Electrical Engineering, Harbin Institute of Technology, lurengui@hit.edu.cn \\ ${ }^{3}$ Department of Electrical Engineering, Harbin Institute of Technology, tch_wang@hit.edu.cn \\ ${ }^{4}$ Department of Electrical Engineering, Harbin Institute of Technology, zhuchunbo@hit.edu.cn
}

\begin{abstract}
It has been shown that none of any energy sources which own high specific energy or high specific power, but not both, can solely fulfil all the demands of hybrid electric vehicle (HEV) in some circumstances. In this paper a simulation model for battery/ultra-capacitor hybrid energy storage system (B/UC HESS) was presented by Matlab/Simulink. Based on the model a low-pass filtering control strategy which adopts ultra-capacitor as load leveling device was developed with a goal of improving battery life. The simulation results under different urban driving cycles show the validity of the model. The peak charge/discharge currents are smoothed effectively, which benefits the battery lifetime improvement. The experiment results also show that the buffering effect of ultracapacitor has optimized the charging and discharging processes of Ni-MH battery considerably.
\end{abstract}

\section{Keywords}

HEV, hybrid energy storage, ultra-capacitor, control strategy, modeling

\section{INTRODUCTION}

For electric vehicles, especially HEVs, possibly more important is the specific power, which related to the acceleration and grade performance that the vehicle can sustain. Consequently the energy source are necessary to have the ability of charge and discharge rapidly and efficiently at wide range temperature environment to provide high power during short duration events such as acceleration, hill-climbing as well as regenerative braking [Williamson et al., 2005; Burke, 2007]. Since the existing battery technology can not satisfy the vehicle demands in some extent due to its low specific power, short life and bad performance at low temperature, which resulted in the vehicle performance decreased. Ultra-capacitor with inherently high specific power and long cycle life for rapid and deep discharges, as well as excellent low temperature behavior [Burke, 2007], is considered more promising technology and capable of improving the ability of energy storage system. By combining battery and ultra-capacitor, the high power load could be taken by ultra-capacitor, battery only responsible for steady load. Thus the battery's cycle life could be effectively extended.

The key issue of B/UC HESS for HEVs depends on how the power between the two sources is assigned. The literatures [Jin-uk et al., 2002; Andersson and Groot, 2003; Baisden and Emadi, 2004; Luk and Ro- sario, 2006] proceeded relative studies to improve the performance of energy storage system for HEV or FCEV by introducing ultra-capacitors. In [Jin-uk et al., 2002], an optimal design of energy storage system using ultra-capacitor and modular development of bi-directional DC/DC converter are introduced. However, the algorithm deciding how much electric energy is taken from or charged to the ultra-capacitor bank for parallel type HEV is not involved yet. According to Andersson and Groot [2003], a low-pass filter control strategy was adopted. When the required load current is less than a certain specified value, the ultra-capacitors were charged from the batteries, using a standard proportional controller having a fixed reference voltage. No dynamic controller, handling for example vehicle speed, was designed in his paper however. Baisden and Emadi [2004] validated a control strategy based on selecting three operation modes of a DC-DC converter. The results of the simulation showed the capability of improving the battery's life due to the decreased current output. Luk and Rosario [2006] suggested a state transition mechanism determines the power split ratio of the dual battery/ultracapacitor sources. Present studies show that power control and management of hybrid energy storage system for HEVs is still a challenge up to now.

In this paper, in the interest of improving the battery life, a model of B/UC HESS for a hybrid electric bus developed by First Automobile Works (FAW) in China is built. For an emphasis on the modeling and control of a B/UC HESS, a low-pass filter control strategy is adopted. When different low-pass filter constant is 
chosen, the ultra-capacitor's effect on buffering the battery charging and discharging currents was investigated. Finally, this control methodology was validated by simulation over three urban driving cycles. Moreover a bench test with low power was done.

\section{STRUCTURE AND MODEL OF THE BAT- TERY/ULTRA-CAPACITOR HYBRID ENERGY} STORAGE SYSTEM

The configuration of B/UC HESS provided in this paper is shown in Figure 1. A bi-directional DC-DC converter locates between DC link and the ultra-capacitor branch, while the battery pack is connected with the DC bus directly. In this configuration, the ultra-capacitors can provide instantaneous power during accelerating or climbing hill as well as accept instantaneous regenerative energy during braking and downhill.

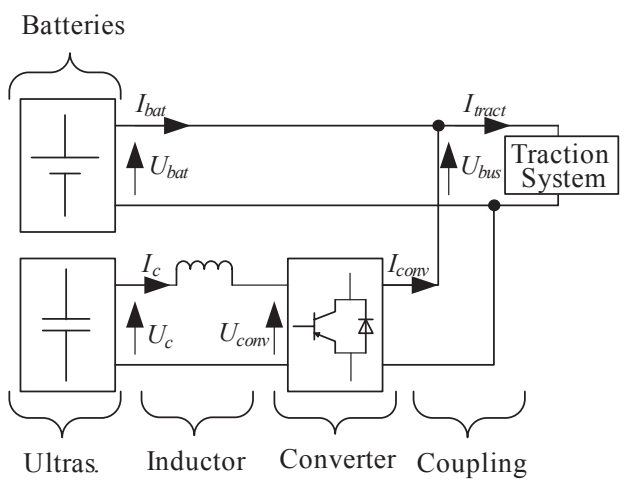

Fig. 1 Scheme of the battery/ultra-capacitor system

\subsection{Battery pack and ultracapacitor pack modeling}

As a key component of hybrid electric vehicles, batteries are devices that transform chemical energy into electrical energy and vice versa. For the battery mod$\mathrm{el}$, an equivalent circuit is adopted, as shown in Figure 2. The relationship of the battery terminal voltage $U_{\text {bat }}$, open-circuit voltage $U_{o c}$, charge/discharge current and internal resistance $\mathrm{R}$ is expressed as (1). $U_{o c}$ is a function of the state of charge (SOC) and temperature. The internal resistances are seen as a function of SOC, current and temperature. The battery SOC is calculated

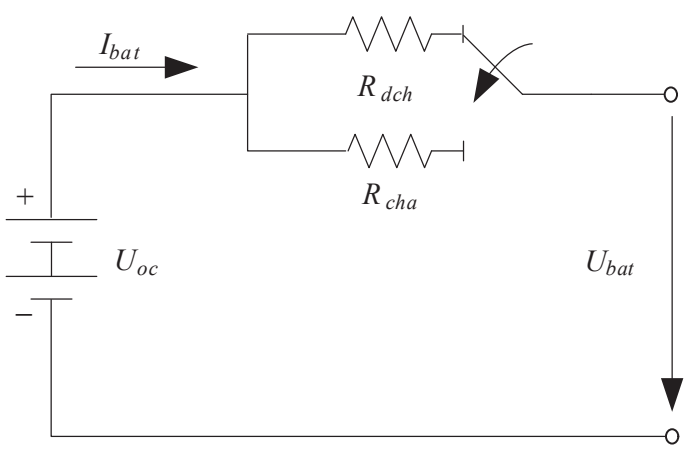

Fig. 2 Battery equivalent circuit by the following equation (2).

$$
\begin{aligned}
& U_{b a t}=U_{o c}-I_{b a t} R \\
& S O C=S O C_{0}+\frac{1}{3600 A_{b a t}} \int i_{b a t} d t
\end{aligned}
$$

Where, $S O C_{0}, A_{b a t}$ and $i_{b a t}$ are the initial value of battery SOC, the battery capacity and current respectively.

Since both the open-circuit voltage and the internal resistance are functions of SOC, the relationships between $U_{o c}, R$ and SOC can be obtained by pulse charge/discharge tests. Figure 3 is $U_{o c}$ vs. SOC curve during the NiMH battery charge and discharge. For NiMH battery, due to a hysteresis phenomena, an average voltage is taken as the open-circuit voltage in battery modeling. The internal resistance vs. SOC curve of one type NiMH battery which used in the real battery model is also presented in Figure 4.

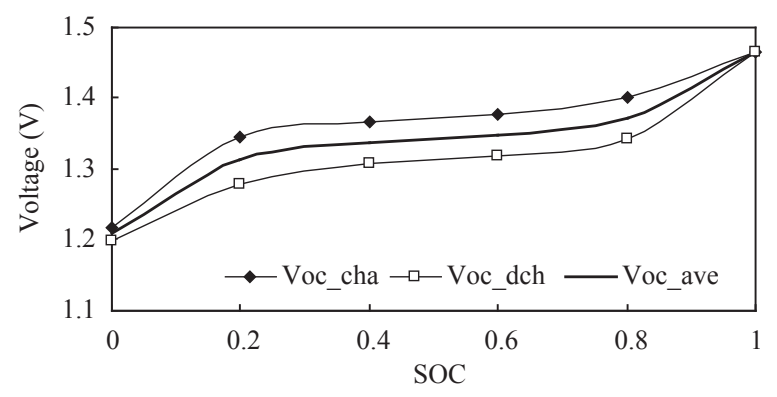

Fig. 3 Uoc vs. SOC curve

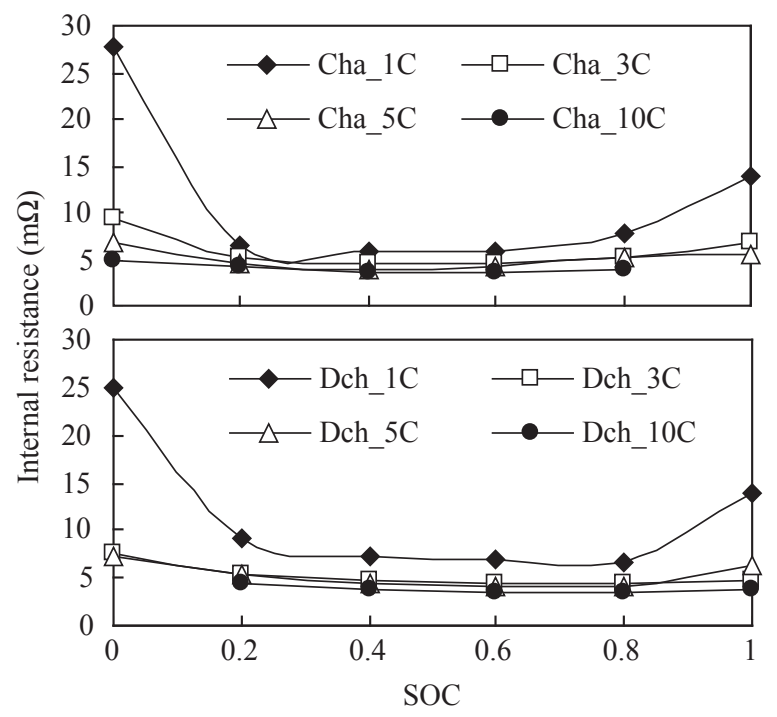

Fig. 4 Internal resistance vs. SOC curve with different current rate

The ultra-capacitor model uses current as an input and limits its operation to the high and low voltage limits. There are three parameters: a capacitance $C$, an equivalent series resistance $R_{i}$ representing the charging 


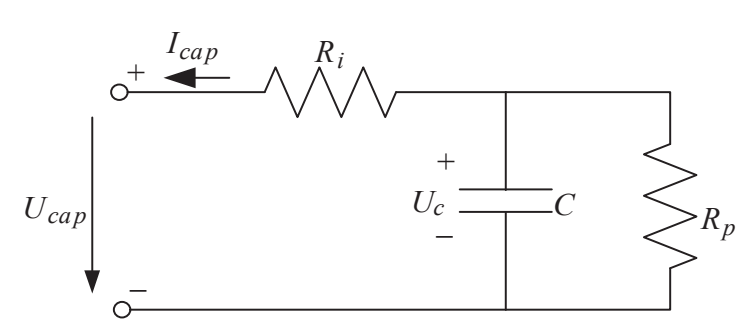

Fig. 5 Ultracapacitor equivalent circuit

and discharging resistance, and an equivalent parallel resistance $R_{p}$ representing the self-discharging losses, shown in Figure 5.

The voltage $U_{c}$ over the capacitance $C$ has the same role as the open-circuit voltage $U_{o}$ of the battery. The capacity voltage $U_{c}$ changes with the electrical charge $Q$. The electrical charge will change in function of the ultra-capacitor current $I_{\text {cell }}$, as expressed in (3) and (4).

$$
\begin{aligned}
& U_{c}=\frac{Q}{C} \\
& Q_{t+1}=Q_{t}-I_{\text {cell }} \Delta t
\end{aligned}
$$

As an energy storage unit of HEVs, ultra-capacitor system will necessarily consist of many cells in series $n_{s}$ to attain the required system voltage and in parallel $n_{p}$. This results in the total capacity voltage $U_{c a p}$ and current $I_{c a p}$, seen from (5) and (6). The internal resistance $R_{i}$ is dependent on the current and temperature. In addition, $R_{p}$ models leakage effects and affects only the long term energy storage performance of ultracapacitors. To simplify the model, its influence is not considered in real modeling. The ultra-capacitor SOC is calculated by the following equation (7).

$$
\begin{aligned}
& U_{\text {cap }}=n_{S} \cdot\left(U_{c}-R_{i} \cdot I_{\text {cell }}\right) \\
& I_{\text {cap }}=n_{p} \cdot I_{\text {cell }} \\
& S O C=\frac{Q_{\text {remaining }}}{C_{\text {total }}}=\frac{C\left(U_{C}-U_{\min }\right)}{C\left(U_{\max }-U_{\min }\right)}=\frac{U_{C}-U_{\min }}{U_{\text {max }}-U_{\min }}
\end{aligned}
$$

\subsection{Power electronics modeling}

\subsubsection{Model of the filtering inductor $L$}

An inductor here has two important functions: store kinetic energy and filter the output voltage. It is expressed by an accumulation element whose state variable is the inductor current $I_{L}$, which depends on the voltage delivered by the ultra-capacitors $U_{c}$ and the output voltage of electric converter $U_{\text {conv }}$.

$$
U_{c}-U_{c o n v}=L \frac{d I_{L}}{d t}+R_{L} I_{L}
$$

Where $L$ is the inductance and $R_{L}$ is the series resistance of the inductor.

The accumulation element needs controller. A current controller generates the reference converter voltage $U_{\text {conv_ref }}$ from the reference current $I_{c_{-} r e f}$.

$U_{\text {conv_ref }}=C_{i}\left[I_{\text {c_ref }}-U_{L_{-} \text {mes }}\right]$

\subsubsection{Model of the electric converter}

The converter (without energy accumulation) is described by the following expressions:

$\left\{\begin{array}{l}U_{\mathrm{conv}}=m U_{b u s} \\ I_{\mathrm{conv}}=m I_{c}\end{array}\right.$

Where $U_{\text {bus }}$ is the DC bus voltage, $I_{\text {conv }}$ and $I_{c}$ represent the output currents of the electric converter and ultracapacitor pack respectively, $m$ is duty cycle.

A pulse width modulation (PWM) can then be used to define the switching functions of the converter.

$m=\frac{1}{U_{\text {bus_mes }}} U_{\text {conv_ref }}$

\subsubsection{Model of the electric coupling}

Electric coupling between the battery pack, the ultracapacitor bank and the traction load part is represented by a parallel electric connection:

$$
\left\{\begin{array}{l}
I_{\text {tract }}=I_{\text {bat }}+I_{\text {conv }} \\
U_{\text {tract }}=U_{\text {conv }}=U_{b u s}
\end{array}\right.
$$

Where $I_{\text {tract }}, I_{b a t}$ represent the currents of the traction load and the battery pack respectively, $U_{\text {tract }}$ is the terminal voltage of the traction load.

\subsection{Traction system modeling}

As the load part of hybrid energy storage system, the traction system is presented by an electric source element. The output current $I_{\text {tract }}$ is obtained from the

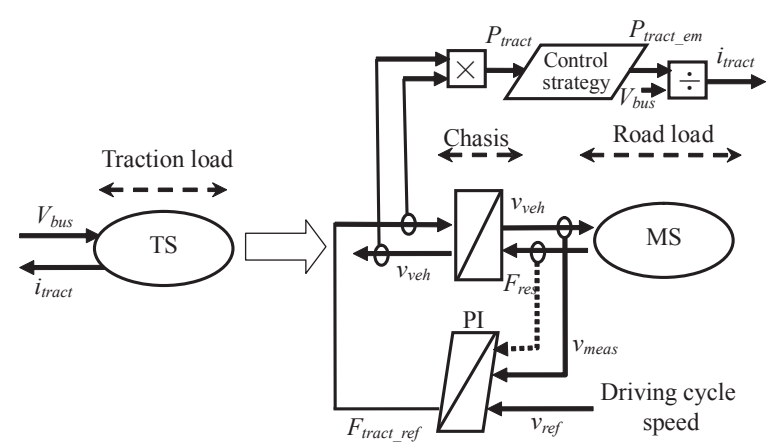

Fig. 6 The model of traction system 
tractive power $P_{\text {tract_em }}$ and the DC bus voltage $V_{\text {bus }}$. And it consists of a chassis and the road environment, as Figure 6 shown. A velocity control has also to be simulated to take all transient condition into account. The reference speed $V_{\text {ref }}$ is deduced from the specific driving cycle.

Chassis is modeled by an accumulation element with the vehicle speed $v_{v e h}$ as the state variable.

$$
F_{\text {tract }}-F_{\text {res }}=M \frac{d}{d t} v_{v e h}
$$

Where $F_{\text {tract }}, F_{\text {res }}$ and $M$ are total tractive effort, the resistance and the mass of the vehicle respectively.

Similarly for the accumulation element, a controller is also required to provide the total effort reference $F_{\text {tract }}$ ref from the velocity measurement $v_{\text {meas }}$ and reference $v_{r e f}$, with a compensation of the resistance $F_{\text {res }}$.

$$
F_{\text {tract_ref }}=C_{v}\left[v_{\text {veh_ref }}-v_{\text {veh_mes }}\right]+F_{\text {res_est }}
$$

Where $C_{v}$ is a velocity controller (PI or IP).

The road environment (MS) is expressed a mechanical source which delivers the resistance to the motion, as (15) shown.

$$
F_{r e s}=M g f \cos +0.047 C_{D} A v^{2}+M g \sin
$$

Where $g$ is the downgrade force, $f$ is rolling resistance coefficient, $C_{D}$ is the aerodynamic coefficient, $A$ is vehicle frontal area, $v$ is vehicle speed, and $\alpha$ is road angle.

Up to now, the model and control of B/UC HESS has been established, shown in Figure 7.

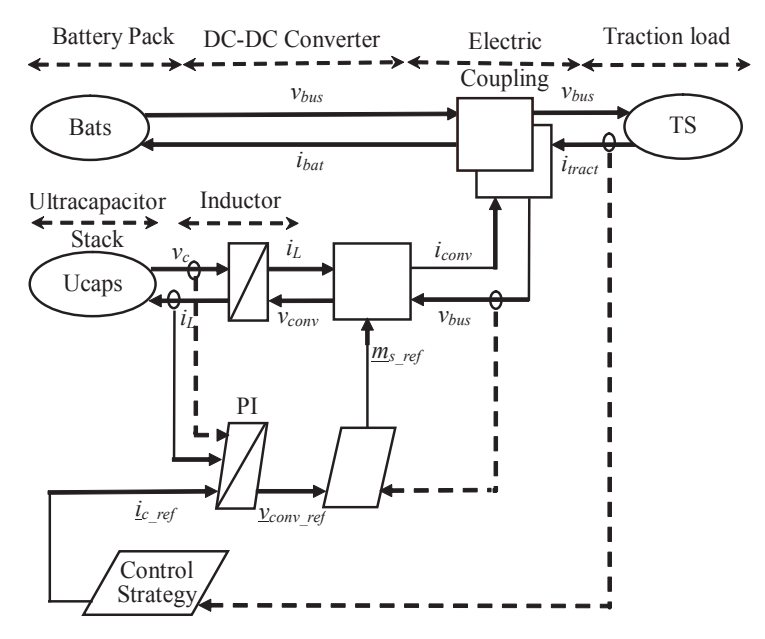

Fig. 7 The model of B/UC HESS

\section{CONTROL STRATEGIES AND METHODS}

Within this study, a control objective is to reduce the peak battery currents for several specific driving cycles. Here a current following control law that par-

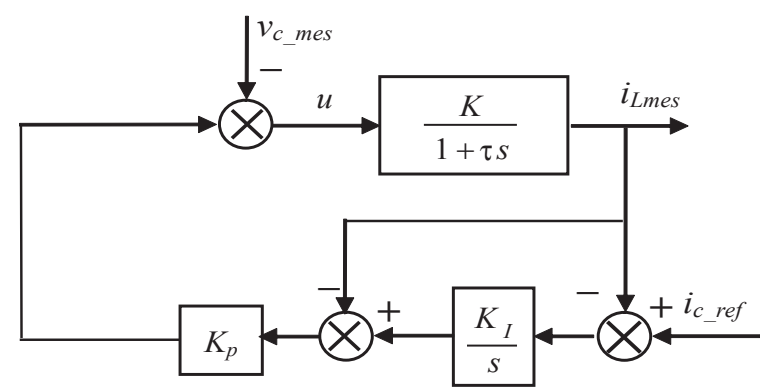

Fig. 8 PI control of the inductor current

titions power distribution between the batteries and ultra-capacitors is addressed. The implement of this control law is shown in Figure 8.

The above control structure allows to control the reference input current of ultra-capacitors, which can be determined by a simple control strategy, namely low-pass filtering method. The main control idea is to maintain the battery current to follow a low-pass filtered current, diverting fast-changing currents to the ultra-capacitors through adjusting the filter time constant. The reference input current of ultra-capacitors is expressed by the following:

$I_{c_{-} \text {ref }}=I_{\text {load }}\left(1-\frac{1}{\tau s+1}\right)$

Where, $\tau$ is the filter time constant.

Restriction condition:

- Current restriction

To guarantee the vehicle runs effectively, the current provided by energy storage system should be equal to the current requirements coming from electric motor:

$$
I_{\text {load }}(t)=I_{b}(t)+m I_{c}(t) \quad t \in(0, T)
$$

Where, $T$ is the vehicle running time.

- SOC restriction

For batteries and ultra-capacitors, to prolong their life, both SOC should be limited within a certain permitted range, namely.

$$
\begin{aligned}
& S O C_{b_{\text {min }}}(t) \leq S O C_{b}(t) \leq S O C_{b_{\text {max }}}(t) \\
& S O C_{c \text { min }}(t) \leq S O C_{c}(t) \leq S O C_{c \text { max }}(t)
\end{aligned}
$$

\section{SIMULATION AND EXPERIMENT RESULTS} The model of B/UC HESS and its control model have been established through Matlab/Simulink environment. Three urban cycles: a modified ECE15-L Urban Cycle, shown in Figure 9, NEDC and BeijingBus driving cycles are chosen to validate the model. Based on 


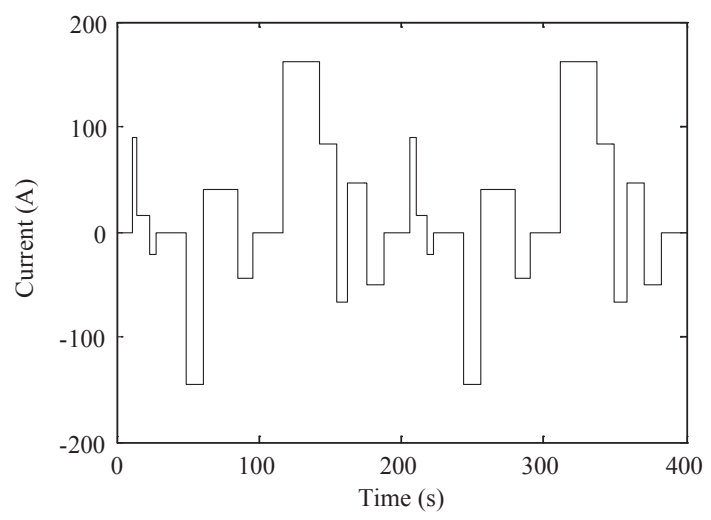

Fig. 9 Modified ECE15-L urban cycle

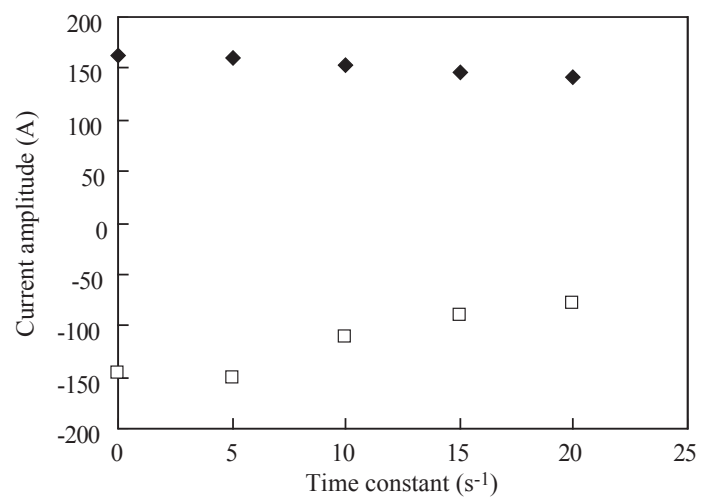

Fig. 10 The battery peak currents with different $\tau$ value

the original Ni-MH batteries, 120 Maxwell PC2500 ultra-capacitors in series are used.

The simulation result of peak current delivered from battery when the filter time constant is different value over modified ECE-15-L urban cycle is shown in Figure 10. As seen in Figure 10, the battery peak charge and discharge currents decrease with the filter time constant increases, especially for peak charge current (corresponding to regenerative braking pattern) resulted from that the role of ultra-capacitors display fully provided that the load current's pulse width is relative narrow. It is obvious that lower filter time constant value will weaken the effect on sharing battery peak current by ultra-capacitors, whereas higher value asks for more single cells resulted in cost and weight increase due to ultra-capacitor's wide voltage range. So $\tau=10$ is chosen in the following simulation.

Figure 11 shows the simulation results both in NEDC and Beijingbus driving cycles. The vehicle speed demands, currents for each energy source compared with that of the original sole battery pack and both battery and ultra-capacitor SOC curves are depicted respectively over above two driving cycles. From the current curves, it is demonstrated that ultra-capacitors have an ability of separating the current segments changing rapidly, which undertakes the burden of batteries.

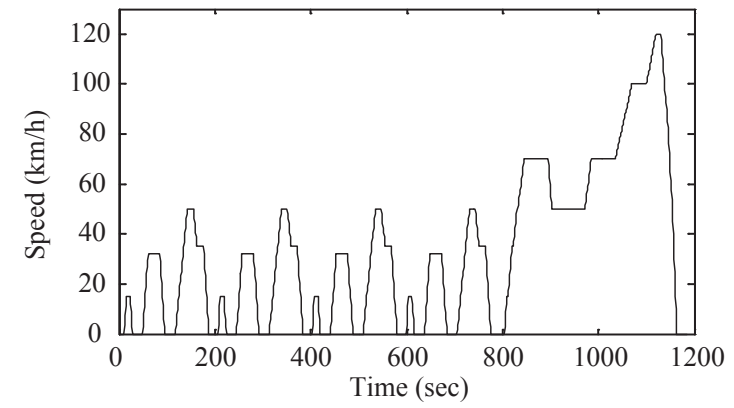

(a) NEDC driving cycle

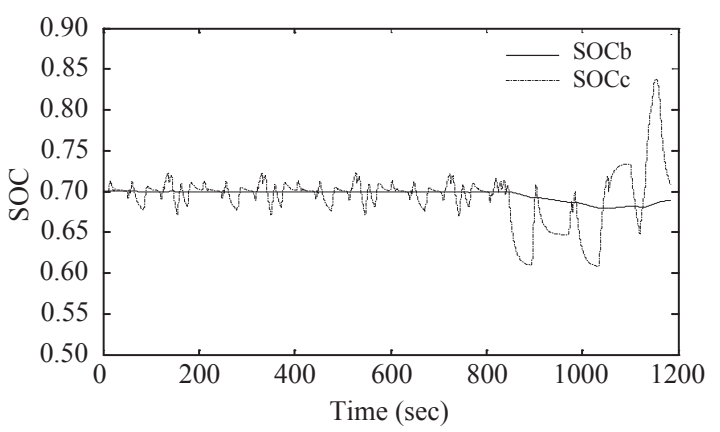

(b) SOC curve over NEDC driving cycle

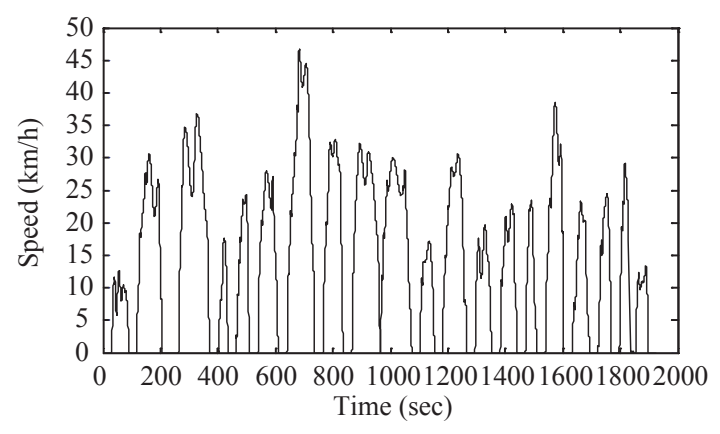

(c) Beijingbus driving cycle

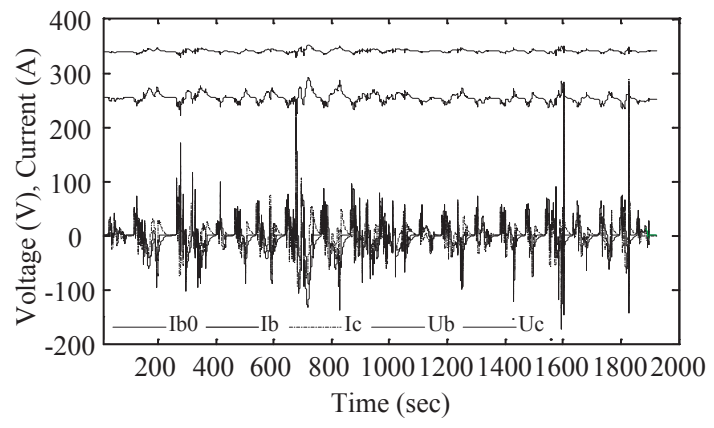

(d) Battery and ultra-capacitor voltage and current curves over Beijingbus driving cycle

Fig. 11 Simulation results

Especially for Beijingbus driving cycle, assisted with ultra-capacitor, the battery peak charge and discharge current is reduced by $30 \%$ and $52 \%$ than the primary battery alone system.

Since a higher DC link voltage rate is required for $\mathrm{HEV}$, the test bench with lower power is built in order to simplify experiment, shown in Figure 12. The DC/ 


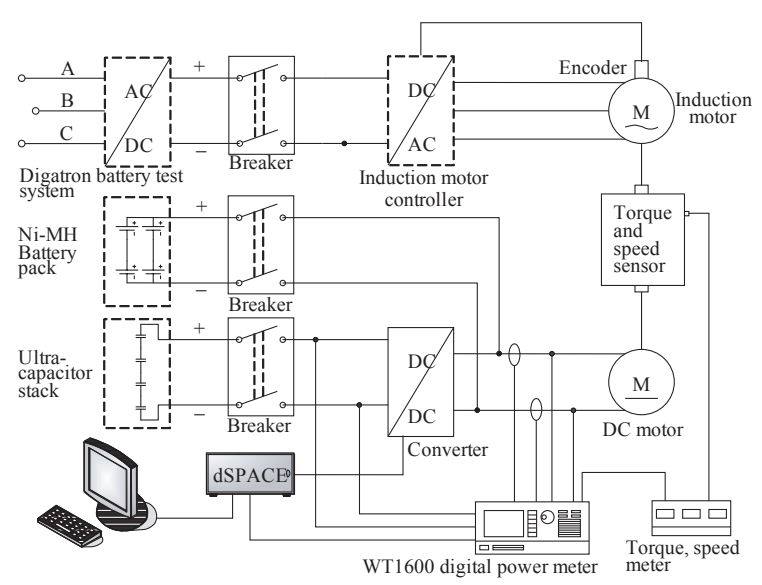

(a) Configuration of the test bench

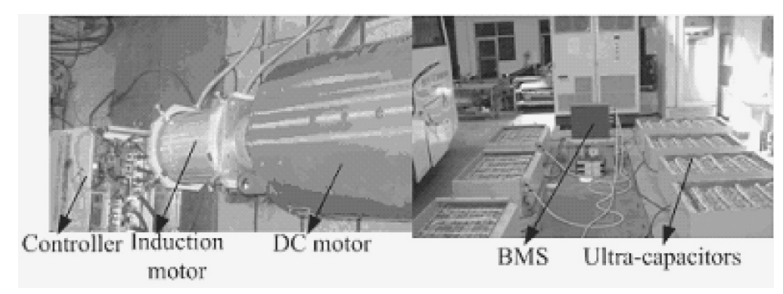

(b) The photo of the real test

Fig. 12 Test bench

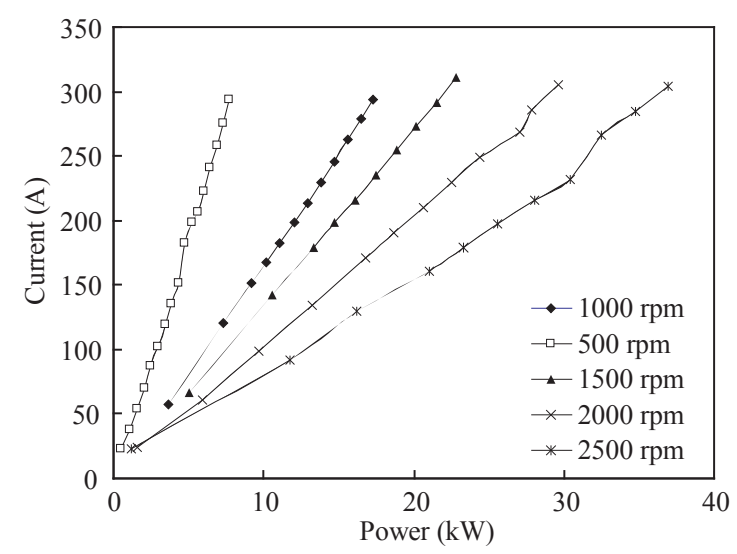

(a) Ultra-capacitor discharge mode

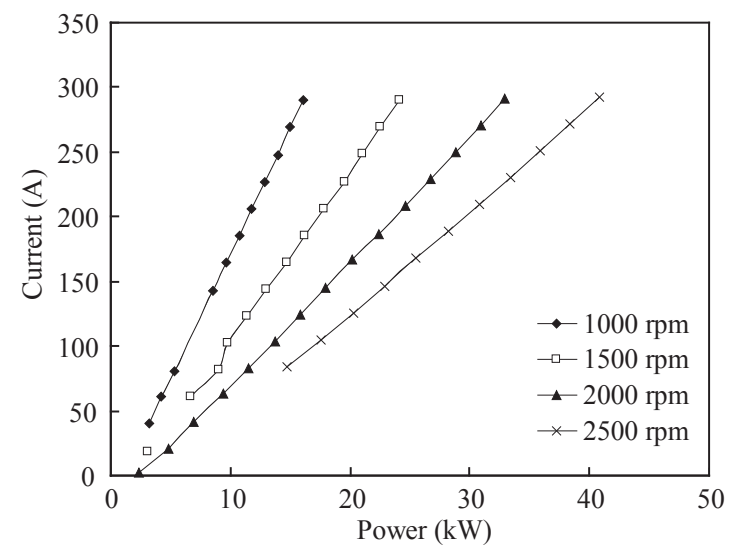

(b) Ultra-capacitor charge mode

Fig. 13 Experiment results when DC/DC converter works at positive and negative modes
DC converter output current under different operating modes are tested when the electric motor runs at $500 \mathrm{rpm}$ to $2500 \mathrm{rpm}$ with 500rpm interval. Figure 13 shows the experiment results. At same rotate speed, the ultra-capacitor charge and discharge current increases consequently with the required power of the electric motor increases, namely a better linear relationship. However at same output power, the current is much lower with rotate speed of electric motor higher due to larger DC link voltage.

\section{CONCLUSION}

Aiming at the electro-chemical battery's low power density and short life in HEV presently, a battery/ ultra-capacitor hybrid energy system is presented in this paper. The study highlights the limitations of traditional batteries, and predicts the battery-ultra-capacitor hybrid energy system would be ideal for HEV over urban driving. The simulation and experiment results both validate that the ultra-capacitors have the ability to reduce peak current demands on the battery pack while satisfying the vehicles power demands and enhance energy recovery during regenerative braking, resulting in longer lifetime for battery.

\section{REFERENCES}

Andersson, T., and J. Groot, Alternative energy storage system for hybrid electric vehicles, Department of Electric Power Engineering Chalmers University of Technology, Master of Science Thesis No. $76 E, 2003$.

Baisden, A. C., and A. Emadi, Advisor-based model of a battery and ultra-capacitor energy source for hybrid electric vehicles, IEEE Transactions on Vehicular Technology, Vol. 53, No. 1, 199-205, 2004.

Burke, A. F., Batteries and ultracapacitors for electric, hybrid, and fuel cell vehicles, Proceedings of the IEEE, Vol. 95, No. 4, 806-820, 2007.

Jin-uk, J., L. Hyeoun-dong, K. Chul-soo, C. Hang-Seok, and C. Bo-Hyung, A development of an energy storage system for hybrid electric vehicles using supercapacitor, 19th Electric Vehicle Symposium, 1379-1388, 2002.

Luk, P. C. K., and L. C. Rosario, Power and energy management of a dual-energy source electric vehicle, Power Electronics and Motion Control Conference, Vol. 1, 1-6, 2006.

Williamson, S. S., A. Khaligh, S. C. Oh, and A. Emadi, Impact of energy storage device selection on the overall drive train efficiency and performance of heavy-duty hybrid vehicles, IEEE Vehicle Power and Propulsion Conference, 381-390, 2005.

(Received March 15, 2010; accepted April 23, 2010) 